\title{
TUBERCULOSIS MULTIDROGORRESISTENTE EN UN HOSPITAL URBANO MARGINAL DE LIMA 2006-2008
}

\author{
Multidrug resistant tuberculosis in a Marginal Urban Hospital in Lima 2006-2008 \\ Víctor Crispín $\mathrm{P}^{1,2}$, Mirtha Roque $\mathrm{A}^{2}$, María E Salazar S², Julio R Ruiz Q ${ }^{2}$
}

${ }^{1}$ Centro Materno Infantil M Grau DISA IV Lima Este. ${ }^{2}$ Instituto de Investigaciones en Química Biológica, Microbiología y Biotecnología "Marco Antonio Garrido Malo" Facultad de Farmacia y Bioquímica - Universidad Nacional Mayor de San Marcos

\section{RESUMEN}

La tuberculosis es un problema de salud pública en el mundoy en el Perú, agravado por las formas multidrogo (TB-MDR) y extensivamente resistentes (TB-XDR). Los objetivos del estudio fueron describir la resistencia a las drogas antituberculosas y los factores de riesgo para TB-MDR en un establecimiento de Salud Urbano Marginal del 2006 al 2008. Es un estudio de reporte de casos realizado en el Hospital de Huaycán, distrito de Ate Vitarte, provincia de Lima. Los datos se obtuvieron del Libro de Registro y Seguimiento de Pacientes con TB-MDR, de las Tarjetas de Control de Tratamiento y de las Historias clínicas. Se reportaron 61 casos de TB-MDR, uno de TB-XDR y 7 monorresistentes; 38 fueron varones ( $55 \%$ ) y 31 mujeres (45\%); el grupo de edad más afectado fue de $15-34$ años con 55 casos (79,6\%). Los factores de riesgo más frecuentes fueron la sospecha de fracaso del esquema uno de tratamiento ( $\mathrm{SF} 1,34$ casos, $49 \%$ ), el fracaso del esquema uno ( $\mathrm{F} 1,14$ casos, $20 \%$ ) yel antecedente de haber sido contacto de un caso de TB-MDR (C-TB-MDR, 12 casos, $17 \%$ ); $76 \%$ de los casos tuvieron resistencia entre 2 a 5 drogas y $13 \%$ entre 6 a 9. Se reportaron 32 patrones diferentes de resistencia a drogas. Todos los casos fueron tratados con esquemas individualizados, 41 casos fueron curados (59,4\%), 16 abandonaron $(23,2 \%)$ y 6 fallecieron $(8,7 \%)$. Se concluye que la TB-MDR afecta a la población joven; entre los factores de riesgo seencuentran la sospecha de fracaso y el fracaso del esquema uno, y el haber tenido contacto con un paciente de TB-MDR; los perfiles de resistencia son múltiplesy variados.

Palabras clave: Tuberculosis-MDR, resistencia bacteriana, patrón de resistencia.

\section{SUMMARY}

Tuberculosis is a public health problem in the world and Peru, aggravated by multidrug (MDR-TB) and extensively resistant (XDR-TB) forms. The objetives were to describe the resistance to tuberculosis drugs and risk factors toacquire MDR-TB in a marginal urban hospital of Lima, since 2006 to 2008. It is a reported cases studyand was undertaken in Huaycán Hospital. The data collected were acquired from the routine reports of the National Control Program and clinical history. A total of 61 cases of MDR-TB, one of XDR-TB and 7 cases of mono resistant tuberculosis were evaluated; 38 were men $(55 \%)$ and 31 women ( $45 \%)$. The more affected age group were 15 to 34 years with 55 cases (79,6\%). The more frecuent risk factors founded to acquire MDR-TB were: suspicion of failure ( $\mathrm{FS} 1,34$ cases, 49\%) and failure of treatment one scheme ( $\mathrm{F} 1$, 14 cases, $20 \%$ ), and to have had contact with MDR-TB patients (MDR-TB-C, 12 cases, $17 \%$ ). $76 \%$ of cases were resistant from 2 to 5 drugs and $13 \%$ from 6 to 9 drugs. There were 32 different resistant patterns to drugs. All cases were treated with individualizad schemes, in 41 cases the treatment was successful $(59.4 \%), 16$ cases abandoned the treatment $(23,2 \%)$ and 6 died $(8,7 \%)$. In conclusion MDR-TB affect young people, the suspicion of failure and failure of treatment and have been in contact with MDR-TB patiens, were the more frecuent risk factors.

Keywords: MDR-Tuberculosis, bacterial resistance, resistance patterns.

\section{INTRODUCCIÓN}

a tuberculosis $(\mathrm{TB})$, enfermedad causada por Mycobacterium tuberculosis, es un problema de larga data para la salud pública mundial y del Perú. Las formas resistentes a drogas de primera y segunda línea empeoran el pronóstico y dificultan su control, obligando a replantear las estrategias sanitarias ${ }^{(1-4)}$.

Se denomina tuberculosis multidrogorresistente (TBMDR) cuando la enfermedad es causada por bacilos resistentes a rifampicina $(\mathrm{R})$ e isoniazida $(\mathrm{H})$, y tuberculosis extensivamente resistente (TB-XDR) cuando presenta resistencia simultánea a las drogas anteriores, a una droga de uso parenteral y a una fluorquinolona ${ }^{(5,6)}$. Sesabequelascepasde M. tuberculosistienen gran variabilidad genética y que la resistencia a drogas se debe a mutaciones cromosomales de la bacteria. Los procedimientos más eficaces para controlar la TB-MDR son el diagnóstico precoz y oportuno, la calificación de la condición del paciente en relación con los factores de riesgo, y el tratamiento con un esquema idóneo basado en la susceptibilidad de la cepa a drogas de primera y segunda líneas, aún cuando la prueba demora entre 60 a 90 días. Es necesario estudiar el perfil de resistencia a drogas del M. tuberculosis en diversos escenarios epidemiológicos sociales, en especial en áreas de elevado riesgo de transmisión de TB como las zonas urbanomarginales, para conocer la naturaleza y extensión del problema, así como los factores de riesgo ${ }^{(5-8)}$.

El estudio tuvo como objetivos determinar los factores de riesgo más frecuentes para enfermar de TBMDR y conocer los patrones de resistencia.

\section{MATERIALES Y MÉTODOS}

Serealizóun estudiodescriptivo, retrospectivoytransversal. La población comprendió a 69 pacientes diagnosti 2006 a 2008 en el Hospital de Huaycán, distrito de AteVitarte, de la Región de Salud IV Lima Este, provincia de Lima. Los criterios de inclusión fueron: ser mayor de 15 años, haber tenido un primer episodio de TB-MDR, no encontrarse en estado de choque ni gravemente desnutrido y no padecer alteraciones congénitas.

Los datos de edad, género, antecedentes de tuberculosis crónica multitratada, abandono del tratamiento, fracaso del tratamiento y la condición de tener o haber tenido contacto con pacientes MDR, fueron obtenidos del Libro de Registro y Seguimiento de pacientes con TB-MDR y de la tarjeta de control 
Tabla 1. Tuberculosis pulmonar resistente a drogas según grupo etario en el Hospital Huaycán 2006-2008.

\begin{tabular}{|c|c|c|c|c|c|}
\hline \multirow{2}{*}{$\begin{array}{l}\text { Grupo } \\
\text { Etario }\end{array}$} & \multicolumn{3}{|c|}{ Año de Estudio } & \multicolumn{2}{|c|}{ Total } \\
\hline & 2006 & 2007 & 2008 & № & $\%$ \\
\hline $15-19$ & 6 & 3 & 2 & 11 & 15,90 \\
\hline $20-24$ & 11 & 3 & 5 & 19 & 27,50 \\
\hline $25-29$ & 2 & 3 & 6 & 11 & 15,90 \\
\hline $30-34$ & 2 & 8 & 4 & 14 & 20,30 \\
\hline $35-39$ & 0 & 0 & 3 & 3 & 4,30 \\
\hline $40-44$ & 1 & 1 & 0 & 2 & 2,90 \\
\hline $45-49$ & 0 & 0 & 1 & 1 & 1,40 \\
\hline $50-54$ & 3 & 0 & 2 & 5 & 7,24 \\
\hline $55-59$ & 1 & 0 & 1 & 2 & 2,90 \\
\hline $60-64$ & 0 & 0 & 0 & 0 & 0,00 \\
\hline $65->$ & 0 & 0 & 1 & 1 & 1,40 \\
\hline Total & 26 & 18 & 25 & 69 & 100.00 \\
\hline
\end{tabular}

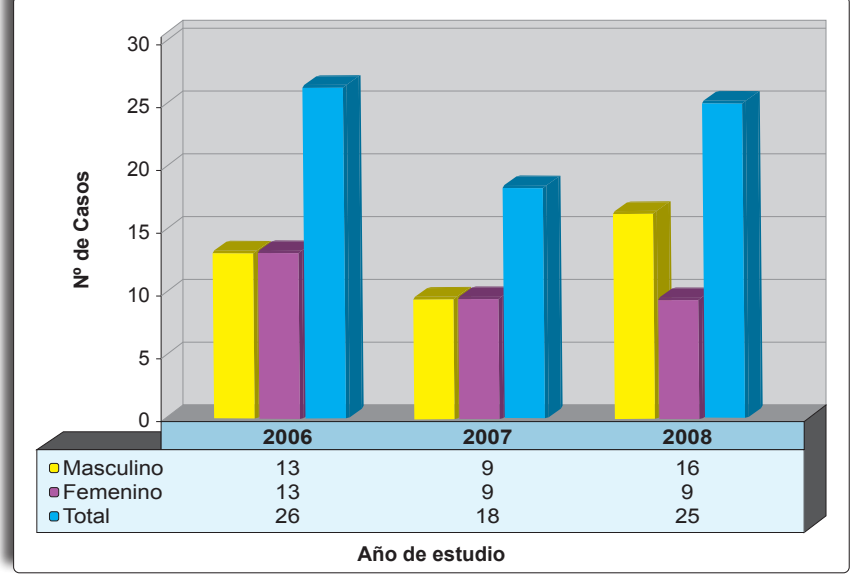

Figura 1. Casos de tuberculosis resistente a drogas según género, Hospital Huaycán 2006-2008.

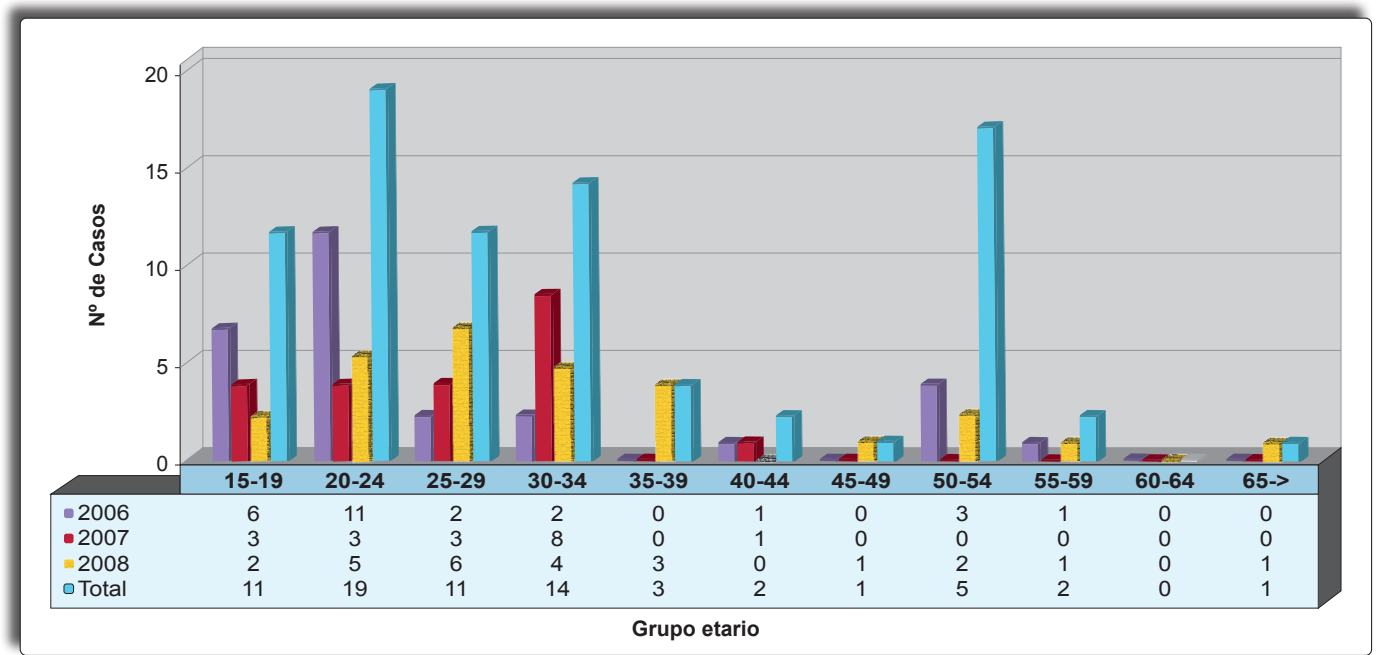

Figura 2. Tuberculosis resistente a drogas según grupo etario en el Hospital Huaycán, 2006-2008.

\section{RESULTADOS} resistente (TB-XDR) y 7 monorresistentes. de población joven (tabla 1, figura 2).

Tabla 2. Tuberculosis pulmonar resistente a drogas según grupo etario en el Hospital huaycán 2006-2008

\begin{tabular}{ccc}
\hline № de drogas & № de casos & \% \\
\hline 1 & 7 & 10,1 \\
2 & 25 & 36,23 \\
3 & 14 & 20,2 \\
4 & 6 & 8,7 \\
5 & 9 & 13,04 \\
6 & 3 & 4,43 \\
7 & 4 & 5,79 \\
8 & 0 & 0 \\
9 & 1 & 1,43 \\
Total & 69 & 100 \\
\hline
\end{tabular}

y hábitos devida no saludables, fueron obtenidos de la historia clínica. Toda la información fue recogida en una ficha ad-hoc. Cuando fue necesario se hizo la visita domiciliaria para complementar información.

Para el análisis de los datos se realizó la digitación en hojas de cálculo Excel versión 3.o y los resultados y gráficos que se muestran se obtuvieron del mismo programa.

Durante el período de estudio fueron identificados 69 pacientes con diagnóstico de tuberculosis resistente a drogas (figura 1), de los cuales 61 correspondieron a la definición de multidrogorresistentes (TB-MDR), uno a extensivamente

Los más afectados fueron 55 pacientes del grupo etario comprendido de 15 a 34 años (79,6\%), observándose que se trata

La sospecha de fracaso del esquema uno (SF1, 49\%), el fracaso del esquema uno $(\mathrm{F} 1,20 \%)$ y haber sido contacto de un caso de TB-MDR (C-TB-MDR, $\quad 17 \%$ ) fueron los factores de riesgo más frecuentes (86\%) (Figura 3).

Los casos monorresistentes fueron el $10 \% ; 76 \%$ expresaron resistencia entre 2 y 5 drogas y $13 \%$ fueron resistentes entre 6 a 9 drogas (tabla 2, figura 4). En los 69 casos estudiados se encontraron hasta 32 patrones diferentes de resistencia a drogas, $13 \%$ de las cepas fueron

de tratamiento. La información sobre la prueba de sensibilidad, comorbilidad inmunosupresora, tratamiento previo particular, tenerfamiliar fallecidoportuberculosis, nivel educativo, ocupación resistentes a $\mathrm{R}$ y $\mathrm{H}$ y $40 \%$ lo fueron a $\mathrm{R}, \mathrm{H}$, y otras drogas. El 10\% de los casos fueron resistentesa $\mathrm{R}, \mathrm{H}$ y kanamicina $(\mathrm{Km})$. Un caso fue identificado como TB-XDR por ser resistente a $R, H$, (drogas 


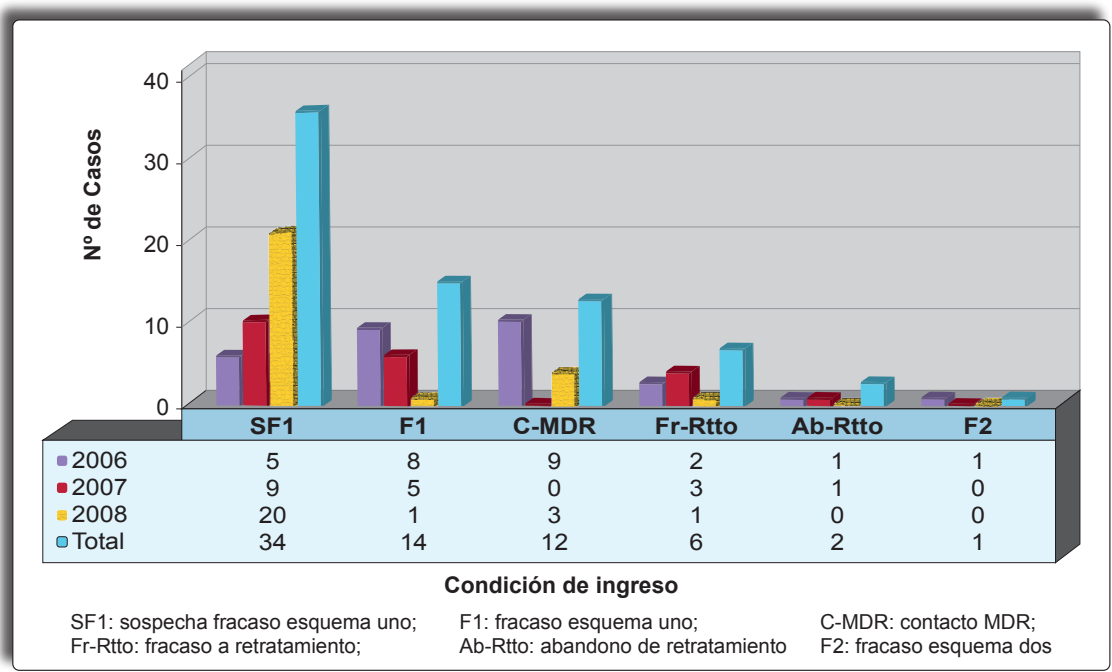

Figura 3. Tuberculosis resistente a drogas según condición de ingreso Hospital Huaycán 2006-2008. fueron diagnosticados en Lima y Callao, especialmente en distritos como San Juan de Lurigancho, San Martín de Porres, La Victoria, Ate, Lima Cercado, El Agustino, Santa Anita, Independenciay Los Olivos ${ }^{(4,5)}$.

El estudio comprendió los años 2006 a 2008 y fueron identificados 69 casos de pacientes con resistencia a drogas antituberculosas (figura 1), de los cuales 61 corresponden a la definición de multidrogorresistentes. Al igual que la TB sensible, la TB-MDR afecta a la población joven, ya que $79,6 \%$ de los pacientes estudiados se encuentran entre las edades de 15 a 34 años (tabla 1), es decir compromete a gran parte de la población económicamente activa. Esta enfermedad, producto de la pobreza, a su vez, genera más de primera línea), Km (droga de segunda línea, inyectable) y ciprofloxacino (fluorquinolona) (tabla 3 ).

Todos los casos fueron tratados con esquemas individualizados, 41 fueron curados $(59,4 \%)$, 16 abandonaron el tratamiento $(23,2 \%)$ y 6 fallecieron $(8,7 \%)$ (tabla 4$)$.

\section{DISCUSIÓN}

Latuberculosis(TB),enfermedad1oo\%curableyprevenible, es un problema muy serio de salud pública por su prevalencia, y sus formas multidrogo (TB-MDR) y extensivamente resistente (TB-XDR), debido a factores demográficos, económicos y sociales. Genera pobreza, ya que el 8o\% de los casos corresponde a la población económicamente activa, siendo los grupos de edad más afectados los comprendidos de 15 a 54 años.

En el Perú desde 1996 hasta el 2007 fueron diagnosticados como TB-MDR 13942 casos y, desde 1999 hasta 2008, entre 186 y 202 casos de TB-XDR. Esta última es producida por cepas mutantes que se han vuelto resistentes a las drogas más efectivas para curar la TB-MDR, como son las fluorquinolonas (ciprofloxacino, ofloxacino, levofloxacino o moxifloxacino) y una de las tres drogas inyectables de segunda línea (Kanamicina, amikacina o capreomicina), loque conviertea las cepas causantes en virtualmente incurables, transmisibles y altamente mortales. La situación de la TB constituye una epidemia de gran magnitud pues 58\% de los casos de TB, 82\% de TB-MDR y $93 \%$ de TB-XDR

Tabla 4. Resultado del tratamiento de tuberculosis resistente a drogas, Hospital Huaycán 2006-2008.

\begin{tabular}{lccccccc}
\hline \multirow{2}{*}{ Resultado } & \multicolumn{3}{c}{ Año de Estudio } & & \multicolumn{2}{c}{ Total } \\
\cline { 2 - 4 } \cline { 6 - 7 } & $\mathbf{2 0 0 6}$ & $\mathbf{2 0 0 7}$ & $\mathbf{2 0 0 8}$ & & No. & \% \\
\hline Curado & 13 & 10 & 18 & & 41 & 59.40 \\
Abandono & 9 & 5 & 2 & & 16 & 23.20 \\
Fallecido & 3 & 2 & 1 & & 6 & 8.70 \\
Fracaso & 0 & 1 & 0 & & 1 & 1.50 \\
Transferido & 1 & 0 & 4 & & 5 & 7.20 \\
Total & 26 & 18 & 25 & & 69 & 100.00 \\
\hline
\end{tabular}

Tabla 3. Patrones de resistencia drogas antituberculosas, Hospital Huaycán 2006-2008.

\begin{tabular}{|c|c|c|c|c|c|}
\hline \multirow{2}{*}{$\begin{array}{c}\text { № } \\
\text { Antibiótico } \\
\end{array}$} & \multirow{2}{*}{$\begin{array}{c}\text { Perfil de } \\
\text { Resistencia }\end{array}$} & \multicolumn{3}{|c|}{ Año de Estudio } & \multirow{2}{*}{ Total } \\
\hline & & 2006 & 2007 & 2008 & \\
\hline \multirow[t]{3}{*}{1} & $\mathrm{H}$ & 1 & 0 & 1 & 2 \\
\hline & S & 2 & 1 & 1 & 4 \\
\hline & Et & 1 & 0 & 0 & 1 \\
\hline \multirow[t]{5}{*}{2} & RH & 6 & 3 & 10 & 19 \\
\hline & $\mathrm{RE}$ & 0 & 0 & 1 & 1 \\
\hline & RS & 1 & 0 & 0 & 1 \\
\hline & $\mathrm{HZ}$ & 0 & 0 & 1 & 1 \\
\hline & HS & 1 & 1 & 1 & 3 \\
\hline \multirow[t]{7}{*}{3} & RHE & 1 & 0 & 1 & 2 \\
\hline & RHZ & 1 & 0 & 0 & 1 \\
\hline & RHS & 2 & 1 & 2 & 5 \\
\hline & RHKm & 1 & 0 & 0 & 1 \\
\hline & RHEt & 0 & 0 & 2 & 2 \\
\hline & RZS & 1 & 0 & 1 & 2 \\
\hline & HSEt & 0 & 0 & 1 & 1 \\
\hline \multirow[t]{5}{*}{4} & RHEZ & 1 & 0 & 1 & 2 \\
\hline & RHZS & 1 & 0 & 0 & 1 \\
\hline & RHEKm & 1 & 0 & 0 & 1 \\
\hline & RHSEt & 0 & 1 & 0 & 1 \\
\hline & HEKmPas & 0 & 1 & 0 & 1 \\
\hline \multirow[t]{4}{*}{5} & RHEZS & 2 & 2 & 1 & 5 \\
\hline & RHEZCX & 1 & 1 & 0 & 2 \\
\hline & RHZSKm & 0 & 1 & 0 & 1 \\
\hline & RHSKmEt & 0 & 0 & 1 & 1 \\
\hline \multirow[t]{3}{*}{6} & RHEZSEt & 1 & 0 & 0 & 1 \\
\hline & RHESKmCm & 0 & 1 & 0 & 1 \\
\hline & RHEZSCx & 0 & 1 & 0 & 1 \\
\hline \multirow[t]{4}{*}{7} & RHESKmCxCm & 0 & 1 & 0 & 1 \\
\hline & RHEZSCxEt & 0 & 1 & 0 & 1 \\
\hline & RHZSCxEtCm & 0 & 1 & 0 & 1 \\
\hline & EZSCxEtCsPas & 1 & 0 & 0 & 1 \\
\hline 9 & RHEZSKmCXEtPas & 0 & 1 & 0 & 1 \\
\hline
\end{tabular}

